Yukawa Institute Kyoto

YITP-96-25

quant-ph./9607012

June 1996

\title{
Generalized Binomial States: Ladder Operator Approach
}

\author{
Hong-Chen $\mathrm{Fu}^{*}$ and Ryu Sasaki \\ Yukawa Institute for Theoretical Physics, Kyoto University, \\ Kyoto 606-01, Japan
}

\begin{abstract}
We show that the binomial states (BS) of Stoler et al. admit the ladder and displacement operator formalism. By generalizing the ladder operator formalism we propose an eigenvalue equation which possesses the number and the squeezed states as its limiting solutions. The explicit forms of the solutions, to be referred to as the generalized binomial states (GBS), are given. Corresponding to the wide range of the eigenvalue spectrum these GBS have as widely different properties. Their limits to number and squeezed states are investigated in detail. The time evolution of BS is obtained as a special case of the approach.
\end{abstract}

\section{Journal of Physics A: Mathematical and General \\ (Accepted for publication)}

\footnotetext{
${ }^{*}$ On leave of absence from Institute of Theoretical Physics, Northeast Normal University, Changchun 130024, P.R.China. E-mail: hcfu@yukawa.kyoto-u.ac.jp
} 


\section{Introduction}

The number and the coherent states of quantized radiation field play important roles in quantum optics and are extensively studied [1]. The binomial states (BS) introduced by Stoler, Saleh and Teich in 1985 [2], interpolate between the most nonclassical number states and the most classical coherent states, and reduce to them in two different limits. Some of their properties [2, 3, 4], methods of generation [2, 3, 5], as well as their interaction with atoms [6], have been investigated in the literature. The BS is defined as a linear superposition of number states in an $M$-dimensional subspace

$$
|\eta, M\rangle=\sum_{n=0}^{M} \beta_{n}^{M}(\eta)|n\rangle,
$$

where $\eta$ is a real parameter satisfying $0<\eta<1$ ("probability"), and

$$
\beta_{n}^{M}(\eta)=\left[\left(\begin{array}{c}
M \\
n
\end{array}\right) \eta^{n}(1-\eta)^{M-n}\right]^{1 / 2} .
$$

The name 'binomial state' comes from the fact that their photon distribution $|\langle n \mid \eta, M\rangle|^{2}=$ $\left|\beta_{n}^{M}(\eta)\right|^{2}$ is simply a binomial distribution with probability $\eta$. In the two limits $\eta \rightarrow 1$ and $\eta \rightarrow 0$ (in both cases "definite probability") it reduces to number states: $|1, M\rangle=|M\rangle$ and $|0, M\rangle=|0\rangle$, respectively. In a different limit of $M \rightarrow \infty, \eta \rightarrow 0$ with $\eta M=\alpha^{2}$ fixed $(\alpha$ real constant), $|\eta, M\rangle$ reduces to the coherent states (not the most general ones, only those with real amplitude $\alpha$ ), which corresponds to the Poisson distribution in probability theory [7]. It is well known that the binomial distribution tends to the Poisson distribution in the above limit [7]. The notion of BS was also generalized to the intermediate number-squeezed states [8] and the number-phase states [9], as well as their $q$-deformation [10].

It is well known that the number and the coherent states are the eigenstates of the number operator $N$ and the annihilation operator $a$, respectively. So we naturally ask if BS is an eigenstate of a proper linear combination of the number operator and the (densitydependent) annihilation operator, or in other words, if it admits a ladder operator definition. The answer is positive. In Sec. 2 we show that BS is the eigenstate of the combination of number operator $N$ and raising operator $J_{M}^{+}=\sqrt{M-N} a$ of $\mathrm{SU}(2)$ via its Holstein-Primakoff realization. This ladder operator formalism enables us to easily derive their displacement operator formalism. The result shows that BS is in fact a special $\mathrm{SU}(2)$ coherent states, as noted in [10].

In Sec. 3, we generalize the ladder operator approach of BS towards the generalized binomial state $(\mathrm{GBS})$ in the sense that they reduce to the number and coherent, squeezed states 
in certain limits. Recall that the squeezed states of the radiation field are the eigenstates of a linear combination of its creation and annihilation operators. So we replace the linear combination of raising and lowering operators of $\mathrm{SU}(2)$ instead of the raising operator in the ladder operator form of binomial states and thus obtain an eigenvalue equation of proper linear combination of all generators of SU(2) (to be referred to as GBS equation for convenience). The GBS equation is exactly solved using a method developed in the investigation of the squeezed states of $\mathrm{SU}(1,1)$ algebras [13] and its $M+1$ distinct eigenvalues and corresponding eigenstates are found. In Sec. 4 we show that these solutions degenerate to the number, coherent and squeezed states in different limits. In Sec. 5 we point out that BS and its time evolution are the special case of GBS equation with a special eigenvalue. We conclude in Sec. 6.

\section{Ladder operator approach to BS}

Let us first consider the ladder operator formalism of BS. To this end we suppose that the BS is an eigenstate of a linear combination of the number operator $N$ and a density-dependent annihilation operator $f(N) a$, namely,

$$
[\mu N+\nu f(N) a]|\eta, M\rangle=\rho|\eta, M\rangle
$$

where constants $\mu, \nu \rho$ and a function $f(N)$ are to be determined. Taking into account that $\mu=1$ in the limit $\eta \rightarrow 1$ and $\mu=0$ in the coherent state limit $\eta \rightarrow 0$, we can simply choose $\mu=\sqrt{\eta}$. Then, inserting the explicit BS (1.1) into (2.1), we obtain the following equations

$$
\begin{aligned}
& \sqrt{\eta^{M}}(\rho-\sqrt{\eta} M)=0, \\
& \sqrt{1-\eta}(\rho-n \sqrt{\eta})=\nu \sqrt{\eta(M-n)} f(n), \quad(n=0,1, \cdots, M-1),
\end{aligned}
$$

from which we find

$$
\rho=\sqrt{\eta} M, \quad \nu=\sqrt{1-\eta}, \quad f(N)=\sqrt{M-N} .
$$

Substituting these results into (2.1), we obtain the ladder operator formalism of BS

$$
[\sqrt{\eta} N+\sqrt{1-\eta} \sqrt{M-N} a]|\eta, M\rangle=\sqrt{\eta} M|\eta, M\rangle .
$$

It is interesting that the operators appearing in the above equation (2.4) are the well-known Holstein-Primakoff realization of Lie algebra $\mathrm{SU}(2)$ :

$$
J_{M}^{0} \equiv \frac{M}{2}-N, \quad J_{M}^{+} \equiv \sqrt{M-N} a, \quad J_{M}^{-} \equiv a^{\dagger} \sqrt{M-N} .
$$


In terms of the $\mathrm{SU}(2)$ generators, (2.4) is rewritten as

$$
\left[\sqrt{\eta} J_{M}^{0}-\sqrt{1-\eta} J_{M}^{+}\right]|\eta, M\rangle=-\frac{\sqrt{\eta} M}{2}|\eta, M\rangle .
$$

This characterization of the BS in terms of the $\mathrm{SU}(2)$ operators is consistent with the original definition (1.1), (1.2) in the two limits of "definite probability" $\eta \rightarrow 1$ and $\eta \rightarrow 0$ :

$$
N|1, M\rangle=M|1, M\rangle, \quad a|0, M\rangle=0,
$$

respectively. To achieve the coherent state in some limit, we multiply $\sqrt{\eta}$ on both sides of (2.4). Then, considering the limit $M \rightarrow \infty$ and $\eta \rightarrow 0$ with fixed $M \eta=\alpha^{2}$ ( $\alpha$ is a real constant) for finite $n$, we arrive at the equation

$$
a|0, \infty\rangle=\alpha|0, \infty\rangle
$$

which is nothing but the ladder (annihilation) operator definition of coherent state.

Here we would like to remark that the binomial state is only one eigenstate of the operator $\sqrt{\eta} N+\sqrt{1-\eta} J_{M}^{+}$corresponding to the eigenvalue $\sqrt{\eta} M$. This operator generally has $M+1$ eigenvalues and eigenstates since it is in fact an $(M+1) \times(M+1)$ matrix. In Sec. 5 , the complete eigenvalues and eigenstates will be presented.

From the ladder operator form of BS we can easily derive its displacement operator form. For this purpose, we identity $\sqrt{\eta}=\sin r, \sqrt{1-\eta}=\cos r, 0<r<\pi / 2$. Then (2.6) can be rewritten as

$$
\left(\cos r J_{M}^{+}-\sin r J_{M}^{0}\right)|\eta, M\rangle=\frac{1}{2} M \sin r|\eta, M\rangle .
$$

Comparing (2.8) with the atomic coherent states and its ladder operator form円 in [12], we find that the BS can be written as

$$
|\eta, M\rangle=e^{-r\left(J_{M}^{+}-J_{M}^{-}\right)}|0\rangle
$$

So BS can be viewed as a special $\mathrm{SU}(2)$ coherent state, as noted in [10].

\section{$3 \quad$ GBS equation and exact solutions}

On the basis of the above analysis, we shall propose a more general eigenvalue equation, the GBS equation, which possesses the number and the squeezed states as its limiting solutions, and present its exact solutions in this section.

From the discussions in Sec.2 we see that in the limit $\eta \rightarrow 0$ and $M \rightarrow \infty$ with fixed $\eta M=\alpha^{2}, \sqrt{\eta} J_{M}^{+} \rightarrow \alpha a$. In fact, we also have $\sqrt{\eta} J_{M}^{-} \rightarrow \alpha a^{\dagger}$ in the same limit. Recall that

\footnotetext{
${ }^{1}$ Symbols here are different from those in [12]: $J_{M}^{ \pm} \rightarrow J_{\mp}, J_{M}^{0} \rightarrow-J_{z}, M \rightarrow 2 J$ and $|0\rangle \rightarrow|-J\rangle$.
} 
the squeezed states of a single mode radiation field can be defined as the eigenstates of the operator $\mu a+\nu a^{\dagger}$, where two complex numbers $\mu$ and $\nu$ satisfy $|\nu / \mu|<1$. So, to achieve the GBS, we should replace $J_{M}^{-}$in the (2.4) by the operator $\mu J_{M}^{+}+\nu J_{M}^{-}$. (Note that $|\nu / \mu|<1$ was necessary for the convergence of an infinite series for the squeezed state. In the GBS case such a constraint is not necessary since the operators are finite matrices.) In summary, we propose the following equation

$$
\left[\sqrt{1-\eta}\left(\mu J_{M}^{+}+\nu J_{M}^{-}\right)-\sqrt{\eta} J_{M}^{0}\right]|\beta, \delta\rangle=\delta|\beta, \delta\rangle
$$

where $\beta=\{\mu, \nu, \eta, M\}$ and $\mu \neq 0$ without loss of generality.

Equation (3.1) is an eigenvalue equation for an $(M+1) \times(M+1)$-matrix. So, generally speaking, it has up to $M+1$ different eigenvalues. If we expand the state $|\beta\rangle$ in terms of the number states, (3.1) will lead to a recursion relation with three terms, which is difficult to solve in its full generality. Here we shall use a method used in the investigation of the squeezed states of $\operatorname{su}(1,1)$ algebra [13]. We write the state $|\beta\rangle$ in the form

$$
|\beta, \delta\rangle=D(\zeta) \| \beta, \delta\rangle, \quad D(\zeta)=\exp \left(\zeta J_{M}^{+}-\zeta^{*} J_{M}^{-}\right)
$$

where the parameter

$$
\zeta=r e^{i \theta}
$$

will be determined later. Then by making use of the following relations

$$
\begin{aligned}
& D^{-1}(\zeta) J_{M}^{+} D(\zeta)=J_{M}^{+} \cos ^{2} r-J_{M}^{-} \sin ^{2} r e^{-2 i \theta}-J_{M}^{0} \sin (2 r) e^{-i \theta} \\
& D^{-1}(\zeta) J_{M}^{-} D(\zeta)=J_{M}^{-} \cos ^{2} r-J_{M}^{+} \sin ^{2} r e^{2 i \theta}-J_{M}^{0} \sin (2 r) e^{i \theta}, \\
& D^{-1}(\zeta) J_{M}^{0} D(\zeta)=\frac{1}{2}\left(J_{M}^{+} e^{i \theta}+J_{M}^{-} e^{-i \theta}\right) \sin (2 r)+J_{M}^{0} \cos (2 r),
\end{aligned}
$$

we obtain an equation for $\| \beta, \delta\rangle$

$$
\left.\left.\left(A_{+} J_{M}^{+}+A_{-} J_{M}^{-}-A_{0} J_{M}^{0}\right) \| \beta, \delta\right\rangle=\delta \| \beta, \delta\right\rangle
$$

where

$$
\begin{aligned}
& A_{+}=\sqrt{1-\eta}\left(\mu \cos ^{2} r-\nu \sin ^{2} r e^{2 i \theta}\right)-\frac{1}{2} \sqrt{\eta} e^{i \theta} \sin (2 r) \\
& A_{-}=\sqrt{1-\eta}\left(\nu \cos ^{2} r-\mu \sin ^{2} r e^{-2 i \theta}\right)-\frac{1}{2} \sqrt{\eta} e^{-i \theta} \sin (2 r) \\
& A_{0}=\sqrt{1-\eta}\left(\mu e^{-i \theta}+\nu e^{i \theta}\right) \sin (2 r)+\sqrt{\eta} \cos (2 r)
\end{aligned}
$$

As an important step, we choose $\zeta$ such that $A_{-}=0$, namely,

$$
\cos ^{2} r\left[\sqrt{1-\eta}\left(\nu-\mu \tan ^{2} r e^{-2 i \theta}\right)-\sqrt{\eta} \tan r e^{-i \theta}\right]=0
$$


where we have used the fact $\cos r \neq 0$ (otherwise, $A_{-}=-\sqrt{1-\eta} \mu e^{2 i \theta} \neq 0$ ). So $\zeta$ is determined by

$$
\mu \sqrt{1-\eta} \Delta^{2}+\sqrt{\eta} \Delta-\sqrt{1-\eta} \nu=0, \quad \Delta=e^{-i \theta} \tan r
$$

and in this case 3.5 is reduced to

$$
\left.\left.A_{+} J_{M}^{+} \| \beta, \delta\right\rangle=\left(\delta+A_{0} J_{M}^{0}\right) \| \beta, \delta\right\rangle
$$

Let $\| \beta, \delta\rangle=\sum_{n=0}^{M} C_{n}|n\rangle$ and insert it into (3.9). We obtain

$$
\begin{aligned}
& C_{M}\left(\delta-M A_{0} / 2\right)=0, \\
& C_{n+1} \sqrt{(n+1)(M-n)} A_{+}=C_{n}\left(\delta+A_{0} M / 2-A_{0} n\right), \\
& \quad(n=0,1, \cdots, M-1) .
\end{aligned}
$$

From (3.10) we have two possibilities: $\delta-M A_{0} / 2=0$ or $C_{M}=0$. In the first case, we can determine one eigenvalue $\delta_{M}=M A_{0} / 2$ and its corresponding eigenstate from (3.11). If $C_{M}=0$, we still have two possibilities: $\delta+A_{0} M / 2-A_{0}(M-1)=0$ or $C_{M-1}=0$. Performing the analysis in the same way, we obtain all eigenvalues and eigenstates. In the general case, $C_{M}=\cdots=C_{k+1}=0$ and $C_{k} \neq 0, k=0,1, \cdots, M,(3.10)$ and (3.11) reduce to

$$
\begin{aligned}
& C_{k}\left(\delta+M A_{0} / 2-A_{0} k\right)=0, \\
& C_{n+1} \sqrt{(n+1)(M-n)} A_{+}=C_{n}\left(\delta+A_{0} M / 2-A_{0} n\right), \\
& \quad(n=0,1, \cdots, k-1) .
\end{aligned}
$$

From (3.12) we obtain the eigenvalues

$$
\delta_{k}=\frac{1}{2} A_{0}(2 k-M), \quad k=0,1, \cdots, M
$$

which are non-degenerate. Substituting (3.14) into (3.13), we can determine the corresponding eigenstates. We evaluate them for two typical cases:

Case 1. If $A_{+}$is non-vanishing, the corresponding eigenstates are obtained as

$$
\left.\| \beta, \delta_{k}\right\rangle=C_{0} \sum_{n=0}^{k}\left(\begin{array}{c}
k \\
n
\end{array}\right)\left(\begin{array}{c}
M \\
n
\end{array}\right)^{-1 / 2} A_{0}^{n} A_{+}^{-n}|n\rangle .
$$

Those eigenstates of different eigenvalues are linearly independent. Using the identity method developed in [14], we can rewrite the eigenstates $\left.\| \beta, \delta_{k}\right\rangle$ in the exponential form

$$
\left.\| \beta, \delta_{k}\right\rangle=C_{0}^{\prime} \exp \left\{\frac{A_{0}}{A_{+}} \sqrt{\frac{k-N+1}{M-N+1}} J_{k}^{-}\right\}|0\rangle,
$$


where we have used the following identity

$$
\left(f(N) a^{\dagger}\right)^{n}|0\rangle=\left(a^{\dagger}\right)^{n} f(1) f(2) \cdots f(n)|0\rangle \quad \text { with } \quad f(N)=\frac{k-N+1}{\sqrt{M-N+1}} .
$$

Let us discuss the cases $k=0$ and $M$ in more detail. In the case of $k=M$, (3.15) becomes

$$
\left.\| \beta, \delta_{M}\right\rangle=\sum_{n=0}^{M}\left[\left(\begin{array}{c}
M \\
n
\end{array}\right) \eta^{\prime n}\left(1-\eta^{\prime}\right)^{M-n}\right]^{1 / 2} e^{i n\left(\theta_{0}-\theta_{+}\right)}|n\rangle,
$$

where

$$
\eta^{\prime}=\frac{\left|A_{0}\right|^{2}}{\left|A_{0}\right|^{2}+\left|A_{+}\right|^{2}}, \quad A_{0}=\left|A_{0}\right| e^{i \theta_{0}}, \quad A_{+}=\left|A_{+}\right| e^{i \theta_{+}} .
$$

It is obvious that the state (3.18) is a binomial state with a very special phase structure. So the eigenstate $\left|\beta, \delta_{M}\right\rangle$ is finally obtained as

$$
\left.\left|\beta, \delta_{M}\right\rangle=D(\zeta) \| \beta, \delta_{M}\right\rangle
$$

which is the displaced binomial state. In the case of $k=0$, it is easy to see from (3.15) that $\left.\| \beta, \delta_{0}\right\rangle=|0\rangle$ and

$$
\left|\beta, \delta_{0}\right\rangle=D(\zeta)|0\rangle
$$

is a binomial state with a phase (see (2.9)).

Case 2. Next let us consider a very special case $A_{+}=0$. It is obvious that the spectrum is given by (3.14) but the eigenstates are determined by

$$
C_{n}\left(\delta_{k}+A_{0} M / 2-A_{0} n\right)=0, \quad(n=0,1, \cdots, k-1), \quad C_{k} \neq 0,
$$

from which we find that $C_{n}=0(n \neq k)$, namely, $\left.\| \beta, \delta_{k}\right\rangle=|k\rangle$, the number states. Therefore, we finally obtain

$$
\left|\beta, \delta_{k}\right\rangle=D(\zeta)|k\rangle
$$

where $\zeta$ should satisfy (3.8) and an additional equation $\left(A_{+}=0\right)$

$$
\sqrt{1-\eta} \nu \Delta^{* 2}+\sqrt{\eta} \Delta^{*}-\sqrt{1-\eta} \mu=0, \quad \Delta^{*}=e^{i \theta} \tan r .
$$

By comparing (3.8) and (3.24) we find these two equations are simultaneously satisfied if $\eta=1$ or

$$
\mu=\nu^{*}
$$

In next section we shall consider the limit $\eta \rightarrow 1$ in which the binomial states tend to the number states.

In conclusion, we have found that (3.1) has $M+1$ distinct eigenvalues and corresponding linearly independent eigenstates and GBS equation (3.1) finally takes the form

$$
\left[\sqrt{1-\eta}\left(\mu J_{M}^{+}+\nu J_{M}^{-}\right)-\sqrt{\eta} J_{M}^{0}\right]\left|\beta, \delta_{k}\right\rangle=\frac{A_{0}}{2}(2 k-M)\left|\beta, \delta_{k}\right\rangle .
$$




\section{Limit to number and squeezed states}

In this section we discuss the limiting cases of the GBS obtained in the previous section. Let us first consider the limit $\eta \rightarrow 1$. In this case (3.8) requires $\Delta=0$ or $\sin r=0(r=m \pi: m$ integers) and $A_{0} \rightarrow 1$ and (3.25) reduces to

$$
N\left|\mu, \nu, 1, M ; \delta_{k}\right\rangle=k\left|\mu, \nu, 1, M ; \delta_{k}\right\rangle .
$$

Namely, $\left|\beta, \delta_{k}\right\rangle$ goes to the number state $|k\rangle$.

The same conclusion can be reached by a different path. From the disentangling theorem 11

$$
D(\xi)=\exp \left(\xi J_{M}^{+}-\xi^{*} J_{M}^{-}\right)=\exp \left(-\tau^{*} J_{M}^{-}\right) \exp \left[-\ln \left(1+|\tau|^{2}\right) J_{M}^{0}\right] \exp \left(\tau J_{M}^{+}\right),
$$

where $\xi=|\xi| e^{-i \phi}, \tau=e^{-i \phi} \tan |\xi|$, we have $D\left(m \pi e^{i \theta}\right)=1$ for any $m$. Furthermore, from (3.6) we have $A_{+}=A_{-}=0$. So the case 2 in the previous section applies and $\left.\| \beta, \delta_{k}\right\rangle=|k\rangle$. We finally arrive at the same conclusion as (4.1):

$$
\left|\beta, \delta_{k}\right\rangle \stackrel{\eta \rightarrow 1}{\longrightarrow}|k\rangle
$$

Therefore we conclude that the limit to the number states is true not only for the binomial states $(\mu=1, \nu=0$ and $k=M)$ but for the more general GBS equations and all of their eigenstates.

Then we turn to the limit to the coherent and the squeezed states. As before we let $M \rightarrow \infty, \eta \rightarrow 0$ with fixed $\eta M=\alpha^{2}$. However, in the present context we have a whole range of the parameter $k, 0 \leq k \leq M$, whose limit must be specified, too. We consider two simple cases.

Case 1. When $k=K+p$, where $K=M / 2$ for even $M$ and $(M \pm 1) / 2$ for odd $M$, and $p$ is finite. In this case $(2 k-M)$ is a finite integer and $\sqrt{\eta}(2 k-M)$ goes to zero in the limit $\eta \rightarrow 0$. Multiplying both sides of (3.25) by $\sqrt{\eta}$ and then taking the limit $M \rightarrow \infty, \eta \rightarrow 0$ with fixed $\eta M=\alpha^{2}$ and $n$, we arrive at

$$
\left(\alpha\left(\mu a+\nu a^{\dagger}\right)-\frac{\alpha^{2}}{2}\right)\left|\mu, \nu, 0, \infty ; \delta_{k}\right\rangle=0 .
$$

So (4.3) becomes

$$
\left(\mu a+\nu a^{\dagger}\right)\left|\mu, \nu, \infty ; \delta_{k}\right\rangle=\frac{\alpha}{2}\left|\mu, \nu, \infty ; \delta_{k}\right\rangle,
$$

from which we see the state $\left|\mu, \nu, 0, \infty ; \delta_{k}\right\rangle$ is a squeezed state.

Case 2. When $(2 k-M) \propto M$, for example, $k=M-p$ or $k=p$, where $p$ is finite. In these cases, as $\eta \rightarrow 0$ and $M \rightarrow \infty$ for fixed $\eta M=\alpha^{2}, \sqrt{\eta} A_{0}(2 k-M)$ becomes infinite. 
This conclusion is based on the assumption that $A_{0}$ remains finite as $\eta \rightarrow 0$, which is true provided $\nu \neq 0$ :

$$
\begin{aligned}
& A_{0}=2 \sqrt{1-\eta}\left(\mu+\nu e^{2 i \theta}\right) \frac{\Delta}{1+|\Delta|^{2}}+\sqrt{\eta} \cos (2 r) \\
& \Delta=-\frac{1}{2 \mu} \sqrt{\frac{\eta}{1-\eta}} \pm \frac{1}{2 \mu} \sqrt{\frac{\eta}{1-\eta}+4 \mu \nu}
\end{aligned}
$$

(The exceptional case of $\nu=0$ will be discussed in some detail in the next section.) Therefore in these cases the naive $\eta \rightarrow 0$ and $M \rightarrow \infty$ limit does not exist for the GBS. In order to define proper limits in these cases we have to consider the situation in which $\mu$ and $\nu$ are $\eta$ dependent.

\section{The case $\nu=0$ : time evolution of BS}

In this section we consider the special case $\nu=0$, for which no $\mathrm{SU}(2)$ rotation is necessary to make $A_{-}=0$, ie. $D(\zeta)=1$. The eigenvalues and eigenstates are directly obtained from (3.14) and (3.15, 3.16) with $A_{0}=\sqrt{\eta}$ and $A_{+}=\mu \sqrt{1-\eta}$

$$
\begin{aligned}
& \delta_{k}=\frac{1}{2} \sqrt{\eta}(2 k-M), \\
&\left|\beta, \delta_{k}\right\rangle=D_{0} \exp \left\{\mu^{-1} \sqrt{\frac{\eta}{1-\eta}} \sqrt{\frac{k-N+1}{M-N+1}} J_{k}^{-}\right\}|0\rangle \\
& \quad=D_{0}^{\prime} \sum_{n=0}^{k}\left(\begin{array}{c}
k \\
n
\end{array}\right)\left(\begin{array}{c}
M \\
n
\end{array}\right)^{-1 / 2} \sqrt{\eta^{n}(1-\eta)^{k-n}} \mu^{-n}|n\rangle,
\end{aligned}
$$

where $D_{0}$ and $D_{0}^{\prime}$ are normalization constants. In particular, if $\mu=1$, we recover the binomial states for $k=M$ and find all the other eigenstates of the operator in (2.4). All these states for $k \neq M$ are new. If $\mu \equiv|\mu| e^{i \phi} \neq 1$, we shall see that $|\mu|$ is not essential but that its phase is related to the time evolution of the states $\left|\beta, \delta_{k}\right\rangle$. In fact $|\mu|$ dependence in (5.3) can be

absorbed by a new parameter $\bar{\eta}=\eta /(\eta+|\mu|(1-\eta))$, which also satisfies $0<\bar{\eta}<1$. So, without loss of generality, we suppose $|\mu|=1$ in the following. To understand the physical meaning of the phase of the parameter $\mu=e^{i \phi}$, we consider the time evolution of the states (5.3). Suppose that at the initial time $t=0$, the radiation field is in the state (5.3), then at any time $t$, it is in the state $U(t)\left|\beta, \delta_{k}\right\rangle$, where $U(t)=e^{-i H t / \hbar}$ is the evolution operator and $H=\omega(N+1 / 2)$ is the Hamiltonian of the single-mode radiation field. It is obvious that

$$
U(t)\left|\beta, \delta_{k}\right\rangle=D_{0}^{\prime \prime} \sum_{n=0}^{k}\left(\begin{array}{c}
k \\
n
\end{array}\right)\left(\begin{array}{c}
M \\
n
\end{array}\right)^{-1 / 2} \sqrt{\eta^{n}(1-\eta)^{k-n}} e^{-i n(\phi+\omega t / \hbar)}|n\rangle,
$$

from which we see that the phase $\phi$ can be understood as the shift of the origin of the time. 
The state $\left|\beta, \delta_{M}\right\rangle$ is essentially the binomial state. It can also be understood as the $\mathrm{SU}(2)$ coherent states. In fact, it is not difficult to see that

$$
\left|\beta, \delta_{M}\right\rangle=\exp \left(\xi^{\prime} J_{M}^{+}-\xi^{\prime *} J_{M}^{-}\right)|0\rangle
$$

where $\xi^{\prime}=-[\arctan \sqrt{\eta /(1-\eta)}] e^{i \phi}$. From (5.5) we see that the states $(5.3)$ are the general $\mathrm{SU}(2)$ coherent states due to the arbitrariness of $\mu$.

It is easy to see that the special cases discussed in the previous section (i) $k=\frac{M}{2}+p$, (iia) $k=M-p$, (iib) $k=p$, ( $p$ : finite) reduce to the coherent states $\left|\alpha e^{-i \phi} / \sqrt{2}\right\rangle$ (i), $\left|\alpha e^{-i \phi}\right\rangle$ (iia) and to the vacuum $|0\rangle$ (iib), respectively, in the limit $M \rightarrow \infty, \eta \rightarrow 0$ with fixed $\eta M=\alpha^{2}$.

\section{Conclusion}

We have found that, among the three methods for defining the coherent state of the radiation field, the ladder and displacement operator methods can be generalized to the study of the BS. The only exception is the minimum uncertainty method. This is understandable since the $\mathrm{BS}$ is between the most non-classical and the most classical states, not the most-classical state (minimum uncertainty state).

On the basis of the analysis of BS we proposed an GBS equation and solved it exactly. The eigenstates of the GBS equation corresponding to $M+1$ distinct eigenvalues are all obtained and their time evolution is discussed. These states range from the displaced binomial states (for $k=M$ ) to the binomial states (for $k=0$ ). They degenerate to the number states and the coherent and squeezed states in two different limits. The original BS of Stoler et al is only an eigenstate of a special GBS equation $(\nu=0$ and eigenvalue $\sqrt{\eta} M / 2)$.

Further investigation of the statistical and phase properties of the GBS obtained in this paper will be published elsewhere.

This work is supported partially by the grant-in-aid for Scientific Research, Priority Area 231 "Infinite Analysis", Japan Ministry of Education. H. C.F is grateful to Japan Society for Promotion of Science (JSPS) for the fellowship. He is also supported in part by the National Science Foundation of China.

\section{References}

[1] Loudon R 1973 The quantum theory of light (Oxford: Clarendon Press);

Klauder J R and Skagerstam B S 1985 Coherent states-Applications in Physics and 
Mathematical Physics (Singapore: World Scientific);

Zhang W M, Feng D H and Gilmore R 1990 Rev. Mod.Phys. 62867

[2] Stoler D, Saleh B E A and Teich M C 1985 Opt. Acta. 32345

[3] Lee C T 1985 Phys. Rev. 31A 1213

[4] Barranco A V and Roversi J 1994 Phys. Rev. 50A 5233

[5] Dattoli G, Gallardo J and Torre A 1987 J. Opt.Soc. Am. 2B 185

[6] Joshi A and Puri R R 1989 J. Mod. Opt. 36 557;

Moggin M E, Sharma M P and Gavrielides A 1990 ibid. 3799

[7] Feller W 19571957 An Introduction to Probability: Theory and Its Applications Vol.1 2nd ed., (John Wiley)

[8] Baseia B, de Lima A F and da Silva A J 1995 Mod.Phys. Lett. 9B 1673

[9] Baseia B, de Lima A F and Marques G C 1995 Phys. Lett. 204A 1

[10] Fan H Y and Jing S C 1994 Phys. Rev. 50A 1909

[11] Truax D R 1985 Phys. Rev. 31D 1988.

[12] Arecchi F T, Courtens E, Gilmore R and Thomas H 1972 Phys. Rev. 6A 2211.

[13] Bergou J A, Hillery M and Yu D 1991 Phys. Rev. 43A 515;

Gerry C C and Grobe R 1995 Phys. Rev. 51A 4123;

Yu D and Hillery M 1994 Quantum Opt. 6 37;

Fan H Y, Ye X and Xu Z H 1995 Phys. Lett. 199A 131;

Fan H Y and Ye X 1993 Phys. Lett. 175A 387;

Fu H C and Sasaki R 1996 Phys. Rev. 53A 3836 (quant-ph/9604004).

[14] Fu H C and Sasaki R 1996 J. Phys. 29A in press. 